$p=0.002)$, whereas there was a non-significant HIV increase among MSM-T with syphilis (OR: 1.36, $\mathrm{p}=0.086$ ).

Conclusion Overall HIV prevalence declined significantly among MSM-T from round 1 to round 2 in Avahan intervention districts in south India. The lack of decline in presence of syphilis suggests that the latter is an important marker of risky behaviour. These results, in conjunction with those obtained in other groups targeted by the intervention; suggest that Avahan had a major impact on the HIV epidemic in south India.

\section{P3.129 IDENTIFYING RECENTLY ACOUIRED HIV INFECTIONS AMONG NEWLY DIAGNOSED MEN WHO HAVE SEX WITH MEN IN THE NETHERLANDS}

doi:10.1136/sextrans-2013-051184.0588

\begin{abstract}
1.2 J Sane, ${ }^{3} \mathrm{M}$ Koot, ${ }^{4} \mathrm{~T}$ Heijman, ${ }^{3} \mathrm{~B}$ Hogema, ${ }^{4} \mathrm{M}$ van Veen, ${ }^{5} \mathrm{H}$ Götz, ${ }^{4} \mathrm{H}$ Fennema, ${ }^{1} \mathbf{E} \mathbf{0 p}$ de Coul. 'Unit of Epidemiology and Surveillance, RIVM, Centre for Infectious Disease Control, Bilthoven, The Netherlands; ${ }^{2}$ European Programme for Intervention Epidemiology Training (EPIET), European Centre for Disease Prevention and Control (ECDC), Stockholm, Sweden; ${ }^{3}$ Sanquin Blood Supply, Virus Diagnostic Services, Amsterdam, The Netherlands; ${ }^{4}$ Amsterdam Public Health Service, Cluster of Infectious Diseases, Department of Research and STI outpatient clinic, Amsterdam, The Netherlands; ${ }^{5}$ Department of Infectious Disease Control, Municipal Public Health Service RotterdamRijnmond, Rotterdam, The Netherlands
\end{abstract}

Background The number of newly diagnosed HIV infections among men who have sex with men (MSM) has gradually increased in the Netherlands during the past decade. However, the current HIV surveillance system cannot differentiate recent HIV infections from longstanding HIV infections. We determined the proportion of recent HIV infections (RI) and estimated HIV incidence using Recent Infection Testing Algorithm (RITA) among newly diagnosed HIV infections among MSM in Amsterdam and Rotterdam.

Methods Plasma samples $(\mathrm{n}=251)$ collected from newly HIVdiagnosed MSM during 2009-2011 at the STI clinics in Amsterdam and Rotterdam were analysed in the study. To test for recent infections, anti-HIV avidity index (AI) was measured in plasma with Architect HIV Ag/Ab Combo immunoassay. Samples were classified as recent if the AI was $\leq 0.80$. Data on viral load, CD4 count and previous HIV testing were incorporated in the RITA algorithm to minimise false recent infections. HIV incidence and 95\% confidence intervals (CI) were estimated using previously described methods. Results Of the 251 samples from MSM, 83 were classified as recent by the avidity index. Five cases were reclassified as non-recent based on low CD4 count $(n=2)$ and viral load $(n=2)$ and history of HIV infection $(\mathrm{n}=1)$ and thus, 78/251 (31\%) infections were determined as recent on RITA. Proportions of RIs in 2009, 2010 and 2011 were $32 \%, 28 \%$ and $33 \%$, respectively. The estimate for combined incidence was $1.5 \%$ per year (95\% CI 1.17-1.83). No significant changes over time were observed.

Conclusions This study estimated the proportion of RIs and HIV incidence among MSM in the Netherlands using the RITA algorithm for the first time. The proportion of RIs was comparable to similar studies in other European countries such as the UK, which however used different methodology.

\section{P3.130 HIGH PREVALENCE LEVELS OF HIV AND SEXUALLY TRANSMISSIBLE INFECTIONS AMONG MONEY BOYS IN CHINA}

doi:10.1136/sextrans-2013-051184.0589

'L Zhang, 'E P Chow, ${ }^{2} X$ Fu, ' $\mathrm{K}$ I lu, 'D P Wilson. 'The University of New South UK, Kensington, Australia; ${ }^{2}$ Renmin University of China, Beijing, China

Background Commercial sex workers within the population of men who have sex with men (MSM) in China, known as 'money boys' (MBs), are perceived to be at higher risk for HIV and other sexually-transmissible infections (STIs).
Methods We conducted a systematic review and meta-analyses from peer-reviewed literature accessed in two English (PubMed and Embase) and three Chinese databases (CNKI, COVIP, Wanfang data). A data synthesis exercise was carried out to determine the extent and patterns of behaviours and HIV/STI epidemics. Pooled estimates, with 95\% confidence intervals, for each study variable were calculated.

Results Thirty-two eligible articles ( 9 in English and 23 in Chinese) were identified. Our analysis indicated that Chinese MBs are generally young, currently employed, at low literacy levels and highly mobile. The prevalence of HIV, syphilis and co-infection among MBs were estimated to be $6.0 \%$ (4.2-8.5\%), $12.4 \%$ (9.9-15.3\%) and $2.2 \%$ (1.1-4.1\%) over the period of 2004-2011. Level of condom use among MBs is generally higher than the broader MSM population $(69.2 \%$ at last act, and $48.5 \%$ consistently over the past 6 months). One-third of the Chinese MBs identified themselves as bisexual and 8.7\% (5.6$13.5 \%)$ are currently married to a female. Further, $40.9 \%$ (34.5$47.7 \%)$ of $\mathrm{MBs}$ participated in group sex in the past 12 months and $14.8 \%(10.6-20.3 \%)$ concurrently use illicit drugs.

Conclusions HIV/STI epidemics have affected Chinese MBs but the evidence suggests that the extent of infections is not greater than among other MSM in China.

\section{P3.131 TRENDS IN HIV INFECTION SURVEILLANCE DATA AMONG MEN WHO HAVE SEX WITH MEN IN ALBANIA, 1993-2012}

doi:10.1136/sextrans-2013-051184.0590

M Dervishi, S Bino, R Bani, S Oyra, F Balla, R Dudushi. Institute of Public Health, Tirana, Albania

Background Albania is still a low HIV prevalence country, however the recent reports on the growing HIV reported cases per year show an increase of reported HIV among MSM in Albania. Furthermore, the results from the repeated bio-behavioural surveillance studies in 2005 and 2008, and 2011 indicate that high risk behaviours are still present among MSM

Methods During the years 1993 to 2012 our study shows increase of HIV among MSM and their socio-demographic and epidemiological characteristics of this group in Albania. These cases are described by age, place of infection, clinical stage of HIV infection, STI co-infection and source partner. To get the estimated trends over this period we used simple linear regression.

Results During the study period, 57 MSM, aged from 17 to 73 years, were diagnosed with HIV. No significant trends over time in overall median age (36 years) were observed. Most of the MSM or, $70 \%$ (40 cases) were infected in Albania and the rest abroad. In the years 1993-2007, 1 to 3 MSM were diagnosed with HIV each year, while in the years 2007-2012 this increased to between 7 and 9 cases. The proportion of MSM, presenting AIDS make up 33\% (19 cases) and the rest were diagnosed in the HIV stage. $28 \%$ (16) of them were married. STI co-infection was reported in $15.7 \%$ ( 9 cases). The majority of them live in urban area, $64 \%$ of them live in capital city, $7 \%$ of them belong to Roma ethnicity.

Conclusions HIV cases among MSM per year are increasing. Current prevalence of HIV in MSM is higher than the general population (from BIO-BSS conducted). The increase in STI coinfections indicates risky sexual behaviour and a potential to spread both HIV and other sexually transmitted infections so HIV prevention strategies should be targeted on this vulnerable group.

\section{P3.132 COULD HIV SEROSORTING EXPLAIN INCREASES IN SYPHILIS PREVALENCE AMONG MEN WHO HAVE SEX WITH MEN? - A MATHEMATICAL MODELLING STUDY}

doi:10.1136/sextrans-2013-051184.0591 